\title{
Corrigendum: Analysis of self-antigen specificity of islet-infiltrating T cells from human donors with type 1 diabetes
}

Jenny Aurielle B Babon, Megan E DeNicola, David M Blodgett, Inne Crèvecoeur, Thomas S Buttrick, René Maehr, Rita Bottino, Ali Naji, John Kaddis, Wassim Elyaman, Eddie A James, Rachana Haliyur, Marcela Brissova, Lut Overbergh, Chantal Mathieu, Thomas Delong, Kathryn Haskins, Alberto Pugliese, Martha Campbell-Thompson, Clayton Mathews, Mark A Atkinson, Alvin C Powers, David M Harlan \& Sally C Kent

Nat. Med.; doi:10.1038/nm.4203; corrected online 15 November 2016

In the version of this article initially published online, the authors forgot to acknowledge the islet-isolation team at the Diabetes Research Institute, University of Miami. This oversight has been corrected for the print, PDF and HTML versions of this article.

\section{Corrigendum: Zika viral dynamics and shedding in rhesus and cynomolgus macaques}

Christa E Osuna, So-Yon Lim, Claire Deleage, Bryan D Griffin, Derek Stein, Lukas T Schroeder, Robert Omage, Katharine Best, Ma Luo, Peter T Hraber, Hanne Andersen-Elyard, Erwing Fabian Cardozo Ojeda, Scott Huang, Dana L Vanlandingham, Stephen Higgs, Alan S Perelson, Jacob D Estes, David Safronetz, Mark G Lewis \& James B Whitney

Nat. Med.; doi:10.1038/nm.4206; corrected online 19 October 2016

In the version of this article initially published online, Robert Were Omange's name was misspelled in the author list. The original version listed Robert Omage. The error has been corrected in the print, PDF and HTML versions of this article.

\section{Corrigendum: Human antibody repertoire after VSV-Ebola vaccination identifies novel targets and virus-neutralizing IgM antibodies}

Surender Khurana, Sandra Fuentes, Elizabeth M Coyle, Supriya Ravichandran, Richard T Davey Jr \& John H Beigel Nat. Med.; doi:10.1038/nm.4201; corrected online 15 November 2016

In the version of this article initially published online, the authors omitted acknowledging a funding agency and researchers for providing the pseudovirion neutralization assay data. The acknowledgments section has been revised to state, "We thank the US Army Medical Research Institute of Infectious Diseases team, including S. Kwilas, M. Wisniewski and J. Hooper, for providing the pseudovirion neutralization assay data used in this study. The pseudovirion work was funded by the US Department of Defense (DoD) Medical Countermeasures Systems' Joint Vaccine Acquisition Program at Fort Detrick, Maryland. The opinions, interpretations, conclusions and recommendations contained herein are those of the authors and are not necessarily endorsed by the US DoD." The error has been corrected for all versions of this article.

Erratum: Resting-state connectivity biomarkers define neurophysiological subtypes of depression

Andrew T Drysdale, Logan Grosenick, Jonathan Downar, Katharine Dunlop, Farrokh Mansouri, Yue Meng, Robert N Fetcho, Benjamin Zebley, Desmond J Oathes, Amit Etkin, Alan F Schatzberg, Keith Sudheimer, Jennifer Keller, Helen S Mayberg, Faith M Gunning, George S Alexopoulos, Michael D Fox, Alvaro Pascual-Leone, Henning U Voss, BJ Casey, Marc J Dubin \& Conor Liston Nat. Med.; doi:10.1038/nm.4246; corrected online 19 December 2016

In the version of this article initially published online, the abstract contained two typos reading, "Like to other neuropsychiatric disorders,..." and "transcranial-magnetic-stimulation therapy..." . These errors have been corrected in the print, PDF and HTML versions of this article. 